\title{
Research on Backstepping Control of a Kind of Second Order System Without Compensation of Coupling Item
}

\author{
Yuliang Chen ${ }^{1}$, Zijian Lin ${ }^{2, a}$ and Junwei Lei ${ }^{2, a}$ \\ ${ }^{1}$ Department of Ordnance Science and Technology, Naval Aeronautical and Astronautical \\ University, Yantai, China \\ ${ }^{2}$ Department of control engineering, Naval Aeronautical and Astronautical University, Yanti, 264001 \\ aleijunwei@126.com
}

Keyword: PID, Stability, Robustness, Hypersonic Vehicle

\begin{abstract}
For a class of two order systems with single input and non-minimum phase, this paper uses backstepping design method to design the controller and studies the Euler programming problem. But unlike the traditional backstepping design, this paper did not use the method of hinge compensation, but there is an inversion of the differential calculation and the use of computer program approximate calculation method. Finally, the simulation results show that the proposed method is effective.
\end{abstract}

\section{Introduction}

Backstepping control method is also called inverse method and reverse recursion method. The main idea is to decompose the high dimensional nonlinear system into several subsystems. Each subsystem is designed to select a hypothetical control to achieve local control objectives. It is starting from the system furthest and introduces the reverse control input until the launch of the whole system, so as to design a real controller. The design advantages of the system can be structured and systematic to solve the problem, which is convenient to deal with the uncertainty and unknown parameters of the system. What's more, it is also a kind of recursive design method of nonlinear system. The design method of backstepping control, due to its unique structural design process and the ability to deal with the non-matching uncertainties, can be well used in the control system design of the missile, the high speed aircraft and so on.

\section{Model Description}

In the condition of unknown input, the backstepping control method reverses derivation step by step forward from the final result through the expected control results. Finally, the control variables are derived to make the system gradually stable until the method of complete control system design is completed. This section is mainly about the derivation of the formula of the inversion method and the modification of the program. Two order inversion control model is as follows:

$$
\begin{gathered}
\dot{x}_{1}=5 x_{1}+x_{2} \\
\dot{x}_{2}=7 x_{1}+8 x_{2}+u
\end{gathered}
$$

Through the design of $u$ to achieve the purpose of changing the control law. Inversion of control law:

$$
u=-10 e_{2}-\left(7 x_{1}+8 x_{2}-\dot{x}_{2}^{d}\right)
$$

According to the control principle and the assumption of a two - order control model, this section designs the angle of attack tracking inversion controller.

$$
\begin{gathered}
\dot{x}_{1}=5 x_{1}+x_{2} \\
\dot{x}_{2}=7 x_{1}+8 x_{2}+u
\end{gathered}
$$

Through the design of $u$, make $x_{1} \rightarrow x_{1}^{d}$. Deduced: 


$$
e_{1}=x_{1}-x_{1}^{d}
$$

So

$$
\dot{e}_{1}=\dot{x}_{1}-\dot{x}_{1}^{d}=5 x_{1}+x_{2}
$$

Finally deduced:

$$
\dot{e}_{1}=5 x_{1}+x_{2}
$$

Assuming $\dot{e}_{1}=-10 e_{1}$, get $\dot{e}_{1} e_{1}=-10 e_{1}^{2} \leq 0$, then there is $e_{1} \rightarrow 0$

make $x_{2} \rightarrow x_{2}^{d}$, introduce $e_{2}=x_{2}-x_{2}^{d}, \quad x_{2}^{d}=-5 x_{1}-10 e_{1}$, so

$$
\dot{e}_{2}=\dot{x}_{2}-\dot{x}_{2}^{d}=7 x_{1}+8 x_{2}+u-\dot{x}_{2}^{d}
$$

Deduced:

$$
\dot{e}_{2}=\dot{x}_{2}-\dot{x}_{2}^{d}=7 x_{1}+8 x_{2}+u-\dot{x}_{2}^{d}
$$

Because $\dot{e}_{2}=-10 e_{2}$

So

$$
-10 e_{2}=7 x_{1}+8 x_{2}+u-\dot{x}_{2}^{d}
$$

\section{Simulation And Program}

According to the formula, the inversion procedure of the control law is derived as follows: clc; clear; close all;

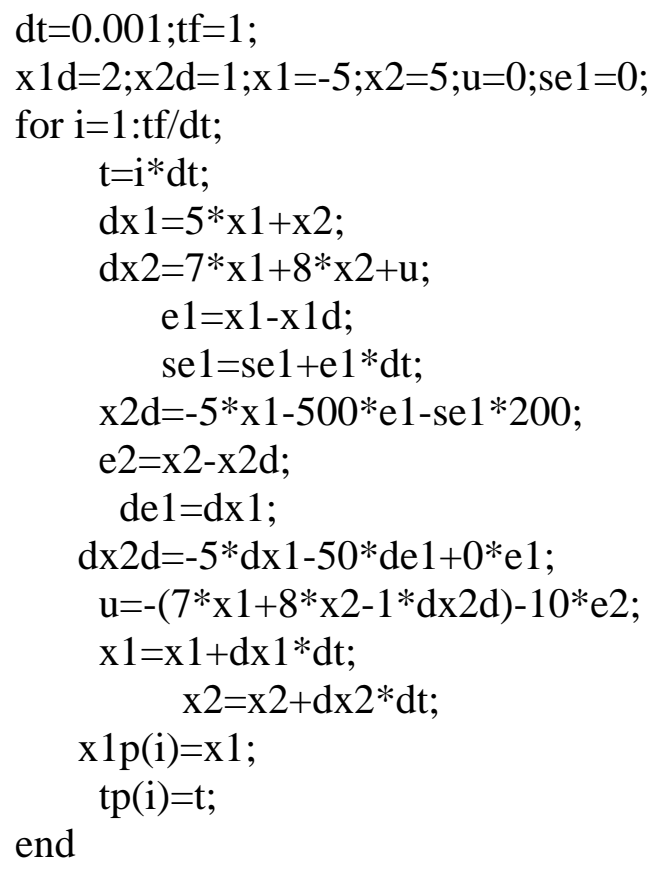

\section{Simulation Results}

According to the above inversion controller design and the inversion procedure, We carried on the digital simulation and obtain the simulation curve as follows: 


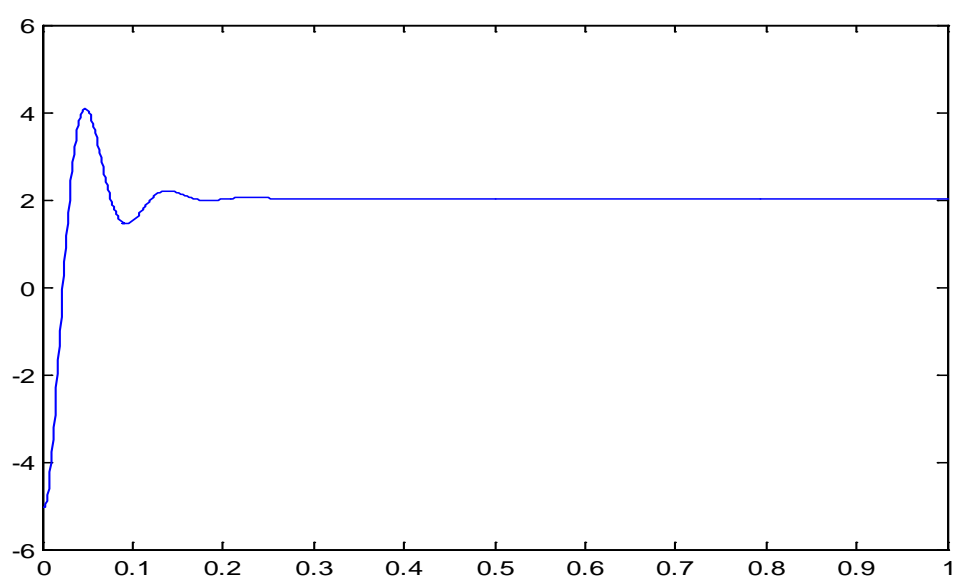

Simulation curve

Through simulation curve, we can see that the control law makes the infinite close to the ideal value and the approach of fast, smooth. The program can not only vividly reflect the rapid and stable system, but also play a role in reducing the reaction time by making the simulation through MATLAB .

\section{Conclusions}

The traditional inversion design takes into account the rigorous problem when the Lyapunov method is used to prove the theory so that the compensation design method of hinge is adopted. But in fact, the compensation is not obvious to the stability of the control system. Therefore this paper designs the inversion controller for second order system without hinges compensate. And by programming and simulation analysis, it shows that without compensation, the system is still able to maintain stable, and has good dynamic performance. Obviously, the inversion controller design method proposed in this paper has the advantages of simple design and easy realization.

\section{References}

[1] F.Sun, Y. Zhao, T. Zhou, Identify fully uncertain parameters and design controllers based on synchronization, Chaos, Solitons and Fractals, 34, (2007)1677-1682

[2] Q. He, L. Wang, B. Liu, Parameter estimation for chaotic systems by particle swarln optimization, Chaos, Solitons and Fractals, 34, (2007)654-661

[3] W. D. Chang, Parameter identification of Chen and Ld systems: A differential evolution approach, Chaos, Solitons and Fractals, 32, (2007)1469-1476

[4] X. Yang, W. Xu, Z. Sun, Estimating model parameters in nonautonomous chaotic systems using synchronization, Physics Letters A, 364, (2007)378-388

[5] L. Shen, M. Wang, Robust synchronization and parameter identification on a class of uncerta. In chaotic systems, Chaos, Solitons and Fractals, 38, (2008)106-111

[6] D. Vassiliadis, Parametric adaptive control and parameter identification of low-dimensional chaotic systems, Physica D, 71, (1994)319-341

[7] J. Lu, J. Cao, Synchronization-based approach for parameters identification in delayed chaotic neural networks, Physica A, 382, (2007)672-682

[8] Y. Yang, X. Ma, H. Zhang, Synchronization and parameter identification of high-dimensional discrete chaotic systems via parametric adaptive control, Chaos, Solitons and Fractals, 28, (2006)244-251 
[9]Bevacqua T, Best E, Huizenga A, and et all, Improved trajectory linearization flight controller for Reusable Launch Vehicles [A], In: 42nd AIAA Aerospace Sciences Meeting and Exhibit, Reno: AIAA, 2004:1-16.

[10]Marwan Bikdash, Ken Sartor, Abdollah Homaifar, Fuzzy guidance of the shuttle orbiter during atmospheric reentry [J], Control Engineering Practice, 1999 (7): 295-303

[11]Wu S. F., Engelen J.H., BabuSka R. Intelligent flight controller design with fuzzy logic for atmospheric reentry Vehicle. AIAA, Aerospace Sciences Meeting and Exhibit, 2000

[12]Austin K. J., Jacobs P.A., Application of Genetic Algorithms to Hypersonic Flight Control[A]. IFSA World Congress and 20th NAFIPS International Conference Austrilia, 2001, 2428-2433

[13]Elmar M. Wallner, Klaus H. Well, Attitude control of a reentry vehicle with internal dynamics [A], In: AIAA Guidance, Navigation, and Control Conference and Exhibit [C], Monterey: AIAA, 2002-4647

[14]S. F. Wu, C. J. H. Engelen, R. Babuska, Fuzzy logic full-envelope autonomous flight control for an atmospheric re-entry spacecraft [J], Control Engineering Practice, 2003 (11): 11-25 